\section{Outpatient Antibiotic Therapy for Osteomyelitis}

To the Editor.-The March 28, 1986 , issue of JAMA contained an article offering an economic analysis of outpatient therapy for osteomyelitis. 'This article is now being distributed by representatives of the Smith Klein \& French pharmaceutical company for the purpose of encouraging the use of the cephalosporin antibiotic cefonicid (Monocid) in this setting. In their introduction, the authors justify their analysis by noting that "a new ... cephalosporin antibiotic, cefonicid sodium, has been shown to be effective in treating osteomyelitis in the outpatient setting." This statement is supported by reference to a clinical trial published in a supplement to Reviews of Infectious Diseases underwritten by Smith Klein \& French. ${ }^{2}$ This clinical trial examined (in an uncontrolled fashion) cefonicid therapy for osteomyelitis in 15 patients, 12 of whom completed therapy. The investigators in this clinical trial appropriately noted the limitations of their study: small numbers, no comparison group, lack of randomization, and infection involving mostly small bones. The authors stated that their data were preliminary and "should serve only as a stimulus for larger and longer studies of a similar nature."

There is no question that there is a need for less costly treatment strategies that involve outpatient intravenous therapy, use of long-acting drugs, and oral therapy. However, trials conducted according to rigorous clinical, epidemiologic, and statistical principles must form the basis for benefit-cost analyses. ${ }^{3,4}$ Cefonicid has yet to be shown to be as effective or safe as standard therapy for the treatment of acute osteomyelitis.

\section{Myron S. Cohen, MD \\ David J. Weber, MD, MPH \\ University of North Carolina} at Chapel Hill

1. Eisenberg JM, Kitz DS: Savings from outpatient antibiotic therapy for osteomyelitis: Economic analysis of a theraotic therapy for osteomyelitis: Economic analys

peutic strategy. JAMA 1986;255:1584-1588. 2. Kunkel MJ, Iannini PB: Cefonidid in a once-daily regimen
for treatment of osteomyelitis in an ambulatory setting. Rev for treatment of osteomyelitis in an a
Infect Dis 1984;6(suppl 4):5865-5869.

Edited by Drummond Rennie, MD, Senior Contributing Editor; Sharon Iverson, Assistant Editor.
3. Koch GG, Sollecito WA: Statistical considerations in the design, analysis, and interpretation of comparative clinical studies. Drug Information J 1984;18:131-151.

Polk $\mathrm{PE}$ Hepler CD: Controversies in antimicrobial . Polk Pharm 1986:43:630-640.

In Reply.-We sympathize with Drs Cohen and Weber's concern that physicians may rely inappropriately on a single scientific report in changing their practices. However, they may have misinterpreted the message of our report on potential savings from outpatient treatment of osteomyelitis. We presented the use of cefonicid as an example of a drug with which "earlydischarge therapy ... may be enhanced." Based on the report by Kunkel and Iannini, ${ }^{1}$ as well as the submission of data for the drug's approval, we came to the same conclusion as the Food and Drug Administration--that cefonicid is an appropriate drug for treatment of many cases of osteomyelitis. Our economic analysis assumed that drug therapy would be started in the hospital and clinical response demonstrated before discharge for outpatient antibiotic therapy. Others have reached similar conclusions, including the authors of a comprehensive review of cefonicid, ${ }^{2}$ who wrote that "patients who require prolonged antibiotic therapy, such as those with osteomyelitis being treated after a relatively short inpatient course, could benefit from the once daily dose given of cefonicid."

We agree that more trials of the longterm safety and efficacy of outpatient antibiotic treatment of serious infections are needed. We would add that these clinical trials should be complemented by rigorous studies of the treatment's efficiency in order to measure financial savings as carefully as clinical benefit.

John M. Eisenberg, MD Deborah S. Kitz, PhD University of Pennsylvania Philadelphia

1. Kunkel MJ, Iannini PB: Cefonicid in a once-daily regimen for treatment of osteomyelitis in an ambulatory setting. $R e$ Infect Dis 1984;6(suppl 4):5865-5869.

2. Saltiel E, Brogden RN: Cefonicid: A review of its antibacterial activity, pharmacologic properties, and therapeutic use Drugs 1986;32:222-259.

\section{Polio Vaccination}

To the Editor.-We were pleased to see the careful analysis of vaccine-associat- ed paralytic poliomyelitis by Nkowane et al. ${ }^{1}$

One important point concerns the estimate of the true risk of vaccineassociated poliomyelitis in susceptible individuals. ${ }^{24}$ This is of more than theoretical interest: in view of the changing epidemiologic situation in developed and developing countries, one has to assume that more and more children being vaccinated for the first time will be fully susceptible to all three poliovirus types. Thus, the rate of vaccineassociated poliomyelitis may be increasing.

Needless to say, this does not call into question the extraordinary blessings of oral poliomyelitis vaccination, particularly in developing countries. But, whenever feasible, consideration should be given to a combined vaccination strategy using inactivated poliomyelitis vaccine for the first polio vaccinations in early infancy, together with diphtheria, tetanus, and pertussis vaccination. This protocol carries the additional advantage of avoiding the possible risk of increasing the incidence of paralytic poliomyelitis by the simultaneous administration of intramuscular injections and of oral polio vaccine. ${ }^{3.5}$

Another lesson to be learned from the contact cases (in these days involving even grandparents) is that vaccination of all household members should be urged, or that at least strict hygienic

\section{Guidelines for Letters}

Letters will be published at the discretion of the editor as space permits and subject to editing and abridgment. They should be typewritten double-spaced and submitted in duplicate. They should not exceed 500 words of text. References, if any, should be held to a minimum, preferably five or fewer. Letters discussing a recent JAMA article should be received within one month of the article's publication. Letters must not duplicate other material published or submitted for publication. An assignment of copyright is essential for publication. It is not feasible routinely to return unpublished letters unless such is requested. Letters not meeting these guidelines are generally not acknowledged. Also see "Instructions for Authors." 